TRANSACTIONS OF THE

AMERICAN MATHEMATICAL SOCIETY

Volume 354, Number 3, Pages 1177-1191

S 0002-9947(01)02917-8

Article electronically published on October 24, 2001

\title{
COMPOSITE BANK-LAINE FUNCTIONS AND A QUESTION OF RUBEL
}

\author{
J. K. LANGLEY
}

Dedicated to the memory of Steve Bank and Lee Rubel

\begin{abstract}
A Bank-Laine function is an entire function $E$ satisfying $E^{\prime}(z)=$ \pm 1 at every zero of $E$. We determine all Bank-Laine functions of form $E=f \circ g$, with $f, g$ entire. Further, we prove that if $h$ is a transcendental entire function of finite order, then there exists a path tending to infinity on which $h$ and all its derivatives tend to infinity, thus establishing for finite order a conjecture of Rubel.
\end{abstract}

\section{INTRODUCTION}

A Bank-Laine function is an entire function $E$ such that $E^{\prime}(z)= \pm 1$ at every zero $z$ of $E$, these arising from differential equations in the following way [1, 12]. Let $A$ be an entire function, and let $f_{1}, f_{2}$ be linearly independent solutions of

$$
w^{\prime \prime}+A(z) w=0,
$$

normalized so that the Wronskian $W=W\left(f_{1}, f_{2}\right)=f_{1} f_{2}^{\prime}-f_{1}^{\prime} f_{2}$ satisfies $W=1$. Then $E=f_{1} f_{2}$ is a Bank-Laine function and

$$
4 A=\left(E^{\prime} / E\right)^{2}-2 E^{\prime \prime} / E-1 / E^{2}, \quad E^{\prime \prime \prime}+4 A E^{\prime}+2 A^{\prime} E=0 .
$$

Conversely, if $E$ is any Bank-Laine function, then [3] the function $A$ defined by (2) is entire, and $E$ is the product of linearly independent normalized solutions of (1). There has been extensive work in recent years concerning the exponent of convergence $\lambda\left(f_{j}\right)$ of the zeros of solutions $f_{j}$, in connection with the order of growth $\rho(A)$ of the coefficient $A$, these are defined by

$$
\lambda\left(f_{j}\right)=\limsup _{r \rightarrow \infty} \frac{\log ^{+} N\left(r, 1 / f_{j}\right)}{\log r}, \quad \rho(A)=\limsup _{r \rightarrow \infty} \frac{\log ^{+} T(r, A)}{\log r} .
$$

In particular it has been conjectured that

$$
A \text { transcendental }, \quad \rho(A)<\infty, \quad \max \left\{\lambda\left(f_{1}\right), \lambda\left(f_{2}\right)\right\}<\infty
$$

implies that $\rho(A)$ is a positive integer: see [1, 20, 23, 24] for partial results in this direction.

There are a number of methods of constructing Bank-Laine functions, although it seems to be relatively difficult to make examples having finite order and associated via (2) with transcendental coefficients. A method observed by Shen [25] uses the Mittag-Leffler theorem: if $\left(a_{n}\right)$ is a complex sequence tending to infinity without

Received by the editors June 12, 2000.

2000 Mathematics Subject Classification. Primary 30D35; Secondary 34M05.

(C)2001 American Mathematical Society 
repetition, then there exists a Bank-Laine function $F$ with zero-sequence $\left(a_{n}\right)$. However, if the sequence $\left(a_{n}\right)$ has finite exponent of convergence, the Bank-Laine function $F$ so constructed may nevertheless have infinite order, and this will always be the case if all the $a_{n}$ are real and $\sum\left|a_{n}\right|^{-1}<\infty$ [15, Theorem 1]. Further methods of construction, which do give rise to Bank-Laine functions $E$ of finite order with $A$ transcendental, were given in 14], using quasiconformal modifications, and in [15], using an elementary variational method.

The investigations of the present paper were prompted by the following question: to what extent is it possible to construct Bank-Laine functions as compositions $E=f \circ g$ of transcendental entire functions $f, g$ ? Examples of composite BankLaine functions include the following:

(a) if $F$ is a Bank-Laine function with $F(0)=0$ and $f(w)=F(w) / w$, then $E(z)=f\left(e^{z}\right)$ is a Bank-Laine function;

(b) if $f$ is an entire function such that $f(a)=0$ implies that $f^{\prime}(a)^{2}\left(1-a^{2}\right)=1$, then $E(z)=f(\sin z)$ is a Bank-Laine function.

Functions $f$ as in (b) may easily be constructed using the Mittag-Leffler theorem. We will prove the following theorem.

Theorem 1.1. Suppose that $f$ and $g$ are non-constant, with $f$ meromorphic in the plane and $g$ entire, and that the composition $E=f \circ g$ is such that $E(z)=0$ implies that $E^{\prime}(z)= \pm 1$. Then one of the following holds.

(i) $f$ has no zeros.

(ii) $f$ has one zero, at $w$, and either $g$ omits the value $w$, or $(g(z)-w) f^{\prime}(w)$ is a Bank-Laine function.

(iii) $f$ has at least two zeros, and $g$ has the form

$$
g(z)=B_{1} z^{2}+B_{2} z+B_{3},
$$

with $B_{1}, B_{2}, B_{3}$ constants. Further, if $f(w)=0$, then $f^{\prime}(w)^{2}\left(B_{2}^{2}-4 B_{1}\left(B_{3}-w\right)\right)=1$.

(iv) $f$ has at least two zeros, and $g$ has the form

$$
g(z)=B_{1} e^{2 b z}+B_{2}+B_{3} e^{-2 b z},
$$

with $b, B_{1}, B_{2}, B_{3}$ constants. Further, if $f(w)=0$, then either $g$ omits the value $w$, or $4 b^{2} f^{\prime}(w)^{2}\left(\left(B_{2}-w\right)^{2}-4 B_{1} B_{3}\right)=1$.

Obviously, if $E$ is entire then $f$ can have at most one pole in Theorem 1.1 The next result shows that Bank-Laine functions of finite order cannot arise as compositions of transcendental functions.

Theorem 1.2. Let $E$ be a Bank-Laine function of finite order. Then $E$ is pseudoprime, that is, $E$ has no factorization of form $E=f \circ g$, with $f, g$ transcendental and $g$ entire, $f$ meromorphic in the plane.

The term pseudoprime comes from the language of factorization theory [7], and there is a substantial literature involving classes of pseudoprime functions. For example, entire functions $F$ with finitely many fixpoints are pseudoprime [5], as are entire functions of finite order with sum of Nevanlinna deficiencies 2 [6]

To prove Theorem 1.1, it suffices to establish the following result.

Theorem 1.3. Let $g$ be an entire function, and let $a_{1}, a_{2}, b_{1}, b_{2}$ be complex numbers with $a_{1} \neq a_{2}$ and $b_{1} b_{2} \neq 0$ and such that $g(z)=a_{j}$ implies that $g^{\prime}(z)= \pm b_{j}$, for $j=1,2$. Then $g$ is a polynomial of degree at most 2 , or has the form (5). 
The proof of Theorem 1.3 requires the following auxiliary result, which has some interest in its own right.

Theorem 1.4. Let $E$ be a Bank-Laine function of finite order, associated via (2) with a transcendental coefficient $A$. Then 0 is an asymptotic value of $E$.

That Theorem 1.4 should hold for $E$ and $A$ as in the hypotheses is perhaps not surprising, given that $E$ is generally small where $A$ is large, by (2), but the difficulties arise from the exceptional set on which the logarithmic derivatives in (2) are large. On the other hand, it would be interesting to know whether it is possible to delete the hypothesis in Theorem 1.4 that $E$ has finite order. We remark that for a non-constant polynomial $A$, it was proved in [13] that there exists a path $\gamma$ tending to infinity on which every solution of (1) tends to 0 , and it would also be interesting to know whether this stronger assertion holds when $A$ is transcendental.

Theorem 1.4 depends on the next result, in which we resolve, for functions of finite order, the following question of Rubel [18, pp. 595-596]: if $f$ is a transcendental entire function, must there exist a path tending to infinity on which $f(z)$ and its derivative $f^{\prime}(z)$ both tend to infinity?

Theorem 1.5. Let $f$ be transcendental and meromorphic in the plane, of order less than $\rho<\infty$, and with finitely many poles. Let $\left(v_{j}\right)$ be a complex sequence such that $v_{j} \rightarrow \infty$ without repetition, and let $n_{0}>0$ be such that

$$
\sum\left|v_{j}\right|^{-n_{0}}<\infty
$$

Let

$$
n_{2}=3 n_{0}+5 \rho+4
$$

Then there exists a path $\gamma$ tending to infinity and not meeting the discs $B\left(v_{j},\left|v_{j}\right|^{-n_{2}}\right)$, such that for each non-negative integer $m$ and each positive real number $c$ we have

$$
\lim _{z \rightarrow \infty, z \in \gamma} \frac{\log \left|f^{(m)}(z)\right|}{\log |z|}=+\infty
$$

and

$$
\int_{\gamma}\left|f^{(m)}(z)\right|^{-c}|d z|<\infty
$$

Here we use the standard notation $B(a, r)$ for the open disc of centre $a$ and radius $r$. If $f$ is any transcendental entire function, the existence of a path $\gamma$ on which $f$ tends to infinity is established by the classical theorem of Iversen, a result substantially strengthened in [10, 17]. Moreover, Rossi proved in [22] that $\gamma$ can be chosen so that $z^{-n} f^{(m)}(z)$ is unbounded on $\gamma$, for every pair of positive integers $m, n$.

\section{LEMMAS NEEDED FOR THE THEOREMS}

Lemma 2.1 ([16]). Suppose that $G$ is transcendental and meromorphic in the plane, of order less than $\rho<\infty$. Then there exists an unbounded uncountable set of positive real numbers $R$ such that the length $L(r, R, G)$ of the level curves $|G(z)|=R$ lying in $|z|<r$ satisfies $L(r, R, G) \leq r^{(4+\rho) / 2}$ for $r \geq \log R$. 
The next lemma is Tsuji's well-known estimate for harmonic measure [27, p.116]. Let $D$ be a domain in the complex plane, regular for the Dirichlet problem. For $0<t<\infty$, define $\theta_{D}^{*}(t)$ to be the angular measure of the intersection of $D$ with the circle $S(0, t)$ of centre 0 and radius $t$, except that $\theta_{D}^{*}(t)=\infty$ if the whole circle $S(0, t)$ lies in $D$.

Lemma $2.2([27])$. Let $z_{0}$ be in $D$, with $2\left|z_{0}\right| \leq r / 2<\infty$, and let $D_{r}$ be the component of $D \cap B(0, r)$ containing $z_{0}$. Then

$$
\omega\left(z_{0}, S(0, r), D_{r}\right) \leq 13 \exp \left(-\pi \int_{2\left|z_{0}\right|}^{r / 2} \frac{d t}{t \theta_{D}^{*}(t)}\right)
$$

Lemma 2.3. Let $0<4 \rho<\sigma<\infty$. Let $z_{0}, z_{1}$ be complex numbers with $\left|z_{1}-z_{0}\right|>$ $\sigma$. Let $U$ be a domain in $\rho<\left|z-z_{0}\right|<\infty$ such that $S\left(z_{0}, t\right)$ meets $\mathbf{C} \backslash U$ for all $t$ with $\rho \leq t \leq \sigma$. Then

$$
\omega\left(z_{1}, S\left(z_{0}, \rho\right), U\right) \leq 26(\rho / \sigma)^{1 / 2}
$$

Proof. Let

$$
w=\phi(z)=\frac{1}{z-z_{0}}, \quad w_{1}=\phi\left(z_{1}\right), \quad V=\phi(U) .
$$

Then $S(0, s)$ meets $\mathbf{C} \backslash V$ for $1 / \sigma \leq s \leq 1 / \rho$. Hence Lemma 2.2 gives

$$
\begin{aligned}
\omega\left(z_{1}, S\left(z_{0}, \rho\right), U\right) & =\omega\left(w_{1}, S(0,1 / \rho), V\right) \\
& \leq 13 \exp \left(-\pi \int_{2 / \sigma}^{1 / 2 \rho} \frac{d t}{t \theta_{V}^{*}(t)}\right) \\
& \leq 13 \exp \left(-\frac{1}{2} \log (\sigma / 4 \rho)\right) \\
& =26(\rho / \sigma)^{1 / 2} .
\end{aligned}
$$

Lemma 2.4. Let $\left(z_{j}\right)$ be a complex sequence such that $z_{j} \rightarrow \infty$ without repetition, and with $\left|z_{j}\right|>2$, and let $N_{1}>0$ be such that

$$
\sum\left|z_{j}\right|^{-N_{1}}<\infty
$$

Let $h$ be transcendental and meromorphic in the plane, of order less than $\rho<\infty$, and with finitely many poles. Let

$$
N_{2}>3 N_{1}+2 \rho
$$

For $m=1,2$, let $H_{m}$ be the union of the closures of the $B\left(z_{j},\left|z_{j}\right|^{-N_{m}}\right)$.

Next, let $R_{1}>4$ be so large that

$$
h^{-1}(\{\infty\}) \subseteq B\left(0, \frac{1}{2} R_{1}\right), \quad M\left(R_{1}, h\right)=\max \left\{|h(z)|:|z|=R_{1}\right\}>e^{4},
$$

and

$$
\log |h(z)| \leq|z / 2|^{\rho} \quad \text { for } \quad|z| \geq R_{1}
$$

and

$$
\left(\frac{1}{2} R_{1}\right)^{N_{2}-N_{1}}>4, \quad \sum_{\left|z_{j}\right|>\frac{1}{2} R_{1}} 26\left|z_{j}\right|^{\rho+\frac{1}{2}\left(N_{1}-N_{2}\right)} \leq \sum_{\left|z_{j}\right|>\frac{1}{2} R_{1}} 26\left|z_{j}\right|^{-N_{1}}<1
$$


Let $w_{0}$ lie outside $H_{1}$, with

$$
\left|w_{0}\right|>R_{1}, \quad\left|h\left(w_{0}\right)\right|>M_{1}^{2}, \quad M_{1}>M\left(R_{1}, h\right)^{2},
$$

and let $C_{0}$ be the component of the set $\left\{z \in \mathbf{C} \backslash H_{2}:|h(z)|>M_{1}\right\}$ in which $w_{0}$ lies.

Let $M>0$. Then there exist arbitrarily large $R$ satisfying

$$
S(0, R) \cap H_{1}=\emptyset,
$$

and such that

$$
\frac{\log ^{+}\left(\sup \left\{|h(z)|: z \in C_{0}, \quad|z|=R\right\}\right)}{\log R}>M .
$$

In particular, $C_{0}$ is unbounded.

Note that in (15) we use (12).

Proof. If $S(0, R) \subseteq C_{0}$ for arbitrarily large $R$ satisfying (17) then the result is obvious. We assume henceforth that $R_{2}>R_{1}$ is such that $S(0, R)$ meets the complement of $C_{0}$ for all $R \geq R_{2}$ satisfying (17).

Let $M_{2}>0$, and assume the existence of an unbounded subset $E_{0}$ of $\left(R_{2}, \infty\right)$ such that $R$ satisfies (17) for all $R$ in $E_{0}$ and such that

$$
\log |h(z)| \leq M_{2} \log |z| \text { for } z \in C_{0}, \quad|z|=R \in E_{0},
$$

such a set $E_{0}$ obviously existing if $C_{0}$ is bounded. Let $R$ be large, in $E_{0}$, and let $D$ be the component of $C_{0} \cap B(0, R)$ in which $w_{0}$ lies.

Let $D=D_{0}$, and form domains as follows. If $D_{m}$ has been formed, choose (if possible) a $z_{j_{m}}$ such that $S\left(z_{j_{m}},\left|z_{j_{m}}\right|^{-N_{2}}\right)$ meets the boundary $\partial D_{m}$ and $S\left(z_{j_{m}}, \lambda_{m}\right) \subseteq$ $D_{m}$ for some $\lambda_{m}$ with $\left|z_{j_{m}}\right|^{-N_{2}} \leq \lambda_{m} \leq\left|z_{j_{m}}\right|^{-N_{1}}$. If such a $z_{j_{m}}$ exists, we set $D_{m+1}=D_{m} \cup B\left(z_{j_{m}}, \lambda_{m}\right)$, while if no such $z_{j_{m}}$ exists, we halt the process. Since $z_{j}$ tends to infinity with $j$, the process must terminate after the formation of some $D_{n}$, and we set $G=D_{n}$.

It follows easily from the construction that $D_{m} \subseteq B(0, R)$ and $\partial D_{m+1} \subseteq \partial D_{m}$ for each $m$. Thus $\partial G$ is contained in a union of $\operatorname{arcs}$ of $S(0, R)$ on which (19) holds, and arcs of level curves $|h(z)|=M_{1}$, as well as arcs of circles $S\left(z_{j},\left|z_{j}\right|^{-N_{2}}\right)$.

Suppose that $S_{j}=S\left(z_{j},\left|z_{j}\right|^{-N_{2}}\right)$ meets $\partial G$. Then $\left|z_{j}\right|>\frac{1}{2} R_{1}$ and $S\left(z_{j}, t\right)$ meets the complement of $G$ for all $t$ with $\left|z_{j}\right|^{-N_{2}} \leq t \leq\left|z_{j}\right|^{-N_{1}}$. Thus Lemma 2.3 and (15) give

$$
\omega\left(w_{0}, S_{j}, G\right) \leq 26\left|z_{j}\right|^{\frac{1}{2}\left(N_{1}-N_{2}\right)} .
$$

To estimate $\omega\left(w_{0}, S(0, R), G\right)$, we note first that $G \subseteq C_{0} \cup H_{1}$. Hence, using (15), we have $\theta_{G}^{*}(t) \leq 2 \pi$ for $R_{2} \leq t \leq R$ and $t \notin E_{1}$, in which $E_{1}$ has measure less than 2. Thus, with $d_{j}$ positive constants independent of $R_{2}$ and $R$, Lemma 2.2 gives

$$
\omega\left(w_{0}, S(0, R), G\right) \leq d_{1} \exp \left(-\pi \int_{\left[2 R_{2}, R / 2\right] \backslash E_{1}} \frac{d t}{t 2 \pi}\right) \leq d_{2}\left(R / R_{2}\right)^{-1 / 2} .
$$

Since (14) gives

$$
\log |h(z)| \leq\left|z_{j}\right|^{\rho} \quad \text { for } \quad\left|z-z_{j}\right| \leq 1, \quad|z| \geq R_{1},
$$


the two-constants theorem and (16), (19), (20) and (21) lead to

$$
\begin{aligned}
2 \log M_{1} & \leq \log \left|h\left(w_{0}\right)\right| \\
& \leq \log M_{1}+\sum_{\left|z_{j}\right|>\frac{1}{2} R_{1}} 26\left|z_{j}\right|^{\rho+\frac{1}{2}\left(N_{1}-N_{2}\right)}+d_{3}\left(R / R_{2}\right)^{-1 / 2} M_{2} \log R,
\end{aligned}
$$

which is plainly impossible if $R$ is large enough, using (13), (15) and (16) again.

\section{Proof of Theorem 1.5}

Take a sequence $S_{k}, k=0,1, \ldots$, such that $f^{-1}(\{\infty\}) \subseteq B\left(0, \frac{1}{4} S_{0}\right)$ and

$$
4<2 S_{k}<S_{k+1}
$$

for each $k$, and such that

$$
\sum_{j=0}^{k} \bar{n}\left(r, 1 / f^{(j)}\right)<r^{\rho}
$$

for $r \geq S_{k}$. For each $k$, let $u_{1, k}, \ldots, u_{\lambda_{k}, k}$ be the distinct points in $S_{k} \leq|z|<S_{k+1}$ at which at least one of $f, \ldots, f^{(k)}$ vanishes, arranged so as to have non-decreasing modulus. Then (23) gives

$$
\sum_{p=1}^{\lambda_{k}}\left|u_{p, k}\right|^{-\rho-1} \leq \sum_{q=1}^{\infty}\left(2^{q} S_{k}\right)^{\rho}\left(2^{q-1} S_{k}\right)^{-\rho-1}=S_{k}^{-1} 2^{\rho+1} .
$$

Arranging the $v_{\mu}$ and $u_{p, k}, 1 \leq p \leq \lambda_{k}, k=0,1, \ldots$, into a sequence $\left(z_{j}\right)$, with $\left|z_{j}\right|$ non-decreasing, it follows from (6), (7), (22) and (24) that

$$
\sum_{j=1}^{\infty}\left|z_{j}\right|^{-n_{1}}<\infty, \quad n_{1}=n_{0}+\rho+1, \quad n_{2}>3 n_{1}+2 \rho .
$$

For $m=1,2$, define

$$
B_{m}=\bigcup_{j=1}^{\infty} B\left(z_{j},\left|z_{j}\right|^{-n_{m}}\right)
$$

and let $H_{m}$ be the union of the corresponding closed discs. We can assume that $\left|z_{j}\right|>2$ and that all $f^{(m)}, m \geq 0$, are non-zero on all of the circles $S\left(z_{j},\left|z_{j}\right|^{-n_{2}}\right)$, decreasing $n_{2}$ slightly, if necessary, to ensure this. Choose a strictly increasing sequence of positive integers $M_{k}$ such that, for each non-negative integer $k$, we have

$$
\left|f^{(k)}(z) / f^{(m)}(z)\right| \leq|z|^{M_{k}} \quad \text { for } \quad 0 \leq m \leq k, \quad|z| \geq S_{k}, \quad z \notin B_{2} .
$$

Such $M_{k}$ exist, by a standard application of the differentiated Poisson-Jensen formula [8, p.22]. Define

$$
g_{k}(z)=f^{(k)}(z) z^{-2 M_{k}},
$$

and choose large $R_{k}$, such that 17 holds for $R=R_{k}$ and

$$
R_{k}>4 S_{k}, \quad M\left(R_{k}, g_{k}\right)>e^{4}, \quad \log \left|g_{k}(z)\right| \leq|z / 2|^{\rho} \quad \text { for } \quad|z| \geq R_{k}
$$

and, using (25),

$$
\sum_{\left|z_{j}\right|>\frac{1}{2} R_{k}} 26\left|z_{j}\right|^{-n_{1}}<(k+1)^{-2}, \quad \sum_{\left|z_{j}\right|>\frac{1}{2} R_{k}} 26\left|z_{j}\right|^{\rho+\frac{1}{2}\left(n_{1}-n_{2}\right)}<1 .
$$


Finally, choose $T_{k}$ using Lemma 2.1, such that all $f^{(m)}, m \geq 0$, are non-zero on the level curves $\left|g_{k}(z)\right|=T_{k}$, and such that

$$
T_{k}>2^{k}, \quad T_{k}>M\left(R_{k}, g_{k}\right)^{2}, \quad L\left(r, T_{k}, g_{k}\right) \leq r^{(4+\rho) / 2} \quad \text { for } \quad r \geq \log T_{k} .
$$

Suppose now that $k$ is a non-negative integer and that $w_{k}$ has been chosen, satisfying

$$
\left|w_{k}\right|>R_{k}, \quad\left|g_{k}\left(w_{k}\right)\right|>T_{k}^{2}, \quad w_{k} \notin H_{1}
$$

such a $w_{k}$ plainly existing for $k=0$. Let $C_{k}$ be the component of the set $\{z \in$ $\left.\mathbf{C} \backslash H_{2}:\left|g_{k}(z)\right|>T_{k}\right\}$ in which $w_{k}$ lies. Then by (29), (301), (31) and Lemma 2.4 there exist an unbounded set $E_{k}$ such that for all $R$ in $E_{k}$ we have (17) and

$$
\sup \left\{\left|g_{k}(z)\right|: z \in C_{k},|z|=R\right\}>R^{2 M_{k+1}} .
$$

In particular $C_{k}$ is unbounded.

Lemma 3.1. There exist arbitrarily large $R$ in $E_{k}$ with

$$
\sup \left\{\left|g_{k+1}(z)\right|: z \in C_{k},|z|=R\right\}>T_{k+1}^{2} .
$$

Proof. This is obviously the case if $S(0, R) \subseteq C_{k}$ for arbitrarily large $R$ in $E_{k}$, and so we assume that $S(0, R)$ meets the complement of $C_{k}$ for all sufficiently large $R$ in $E_{k}$. Let $R$ be large, in $E_{k}$, and using (28) and (33) choose $\zeta_{1}$ in $C_{k}$ with $\left|\zeta_{1}\right|=R$ and

$$
\left|g_{k}\left(\zeta_{1}\right)\right|>R^{2 M_{k+1}}, \quad\left|f^{(k)}\left(\zeta_{1}\right)\right|>R^{2 M_{k}+2 M_{k+1}} .
$$

Follow the circle $|z|=R$ counterclockwise until the first point $\zeta_{2}$ of intersection with $\partial C_{k}$. By (17) and (28) we must have

$$
\left|g_{k}\left(\zeta_{2}\right)\right| \leq T_{k}, \quad\left|f^{(k)}\left(\zeta_{2}\right)\right| \leq T_{k} R^{2 M_{k}} .
$$

If $\left|g_{k+1}(z)\right| \leq T_{k+1}^{2}$ for all $z$ on the open arc $\sigma$ of the circle $S(0, R)$ from $\zeta_{1}$ to $\zeta_{2}$, then integration of $f^{(k+1)}$ and (34) and (35) give

$$
R^{2 M_{k}+2 M_{k+1}} \leq T_{k} R^{2 M_{k}}+2 \pi T_{k+1}^{2} R^{2 M_{k+1}+1},
$$

an obvious contradiction if $R$ is large enough.

We now construct the path $\gamma$. Using Lemma 3.1, choose $w_{k+1}$ in $C_{k} \backslash H_{1}$ such that $\left|w_{k+1}\right|>R_{k+1}$ and $\left|g_{k+1}\left(w_{k+1}\right)\right|>T_{k+1}^{2}$. For each non-negative integer $k$, let $\gamma_{k}$ be a path from $w_{k}$ to $w_{k+1}$ in the closure of $C_{k}$, consisting of part of the ray $\arg z=\arg w_{k}$, part of the circle $S\left(0,\left|w_{k+1}\right|\right)$ and part of the boundary $\partial C_{k}$. We can assume that all the $f^{(m)}, m \geq 0$, are non-zero on the ray $\arg z=\arg w_{k}$ and the circle $S\left(0,\left|w_{k+1}\right|\right)$, adjusting $w_{k}$ and $w_{k+1}$ slightly, if necessary, to achieve this. Since $\left|w_{k}\right|>R_{k}$ and $\left|g_{k}(z)\right| \geq T_{k}>M\left(R_{k}, g_{k}\right)^{2}$ on $\gamma_{k}$, we see that the union of the $\gamma_{k}$ forms a path $\gamma$ which tends to infinity. Also, if $0 \leq m \leq k$, then (27) gives

$$
\left|f^{(m)}(z)\right| \geq|z|^{-M_{k}}\left|f^{(k)}(z)\right|=|z|^{M_{k}}\left|g_{k}(z)\right| \geq T_{k}|z|^{M_{k}},
$$

for $z$ in the closure of $C_{k}$ and, since $T_{k} \rightarrow \infty$ and $M_{k} \rightarrow \infty$, this proves (8).

To prove (9) we assume that $c$ is a positive constant, $m$ is a non-negative integer and that $k$ is a positive integer, large compared to $m$. If a circle $S_{j}=S\left(z_{j},\left|z_{j}\right|^{-n_{2}}\right)$ meets $\gamma_{k}$, then (36) gives

$$
\int_{S_{j} \cap \gamma_{k}}\left|f^{(m)}(z)\right|^{-c}|d z| \leq 2 \pi\left|z_{j}\right|^{-n_{2}} .
$$


Next, the contributions of the ray $\arg z=\arg w_{k}$ and the circle $S\left(0,\left|w_{k+1}\right|\right)$ to the integral $\int_{\gamma_{k}}\left|f^{(m)}(z)\right|^{-c}|d z|$ have sum at most

$$
T_{k}^{-c} \int_{R_{k}}^{\infty} t^{-c M_{k}} d t+2 \pi T_{k}^{-c}\left|w_{k+1}\right|^{1-c M_{k}}<T_{k}^{-c}
$$

if $k$ is large enough. Also, if $T=\left\{z \in \gamma_{k}:\left|g_{k}(z)\right|=T_{k}\right\}$, then (31) and (36) give

$$
\begin{aligned}
\int_{T}\left|f^{(m)}(z)\right|^{-c}|d z| & \\
& \leq T_{k}^{-c}\left(\left(\log T_{k}\right)^{(4+\rho) / 2}+\sum_{q=0}^{\infty}\left(2^{q} \log T_{k}\right)^{-c M_{k}}\left(2^{q+1} \log T_{k}\right)^{(4+\rho) / 2}\right) \\
& \leq 2 T_{k}^{-c}\left(\log T_{k}\right)^{(4+\rho) / 2}
\end{aligned}
$$

if $k$ is large enough. Combining the last estimate with (37) and (38), and using (30), (31) and the fact that $f^{(m)} \neq 0$ on $\gamma$, we obtain (9).

\section{Proof of Theorem 1.4}

Let $E$ and $A$ be as in the hypotheses. We apply Theorem 1.5, with $f=A$ and the $v_{j}$ the points in $|z|>2$ at which $E(z)=0$. Choose $n_{0}>0$ such that (6) holds, and define $n_{2}$ by (17). Standard estimates based on the differentiated Poisson-Jensen formula [8, p.22] give a constant $M>0$ such that

$$
\left|E^{\prime}(z) / E(z)\right|^{2}+\left|E^{\prime \prime}(z) / E(z)\right| \leq|z|^{M}
$$

for all $z$ with $|z|>4$ and lying outside the union of the $\operatorname{discs} B_{j}=B\left(v_{j},\left|v_{j}\right|^{-n_{2}}\right)$. Theorem 1.5 gives a path $\gamma$ tending to infinity and not meeting the $B_{j}$, such that

$$
\lim _{z \rightarrow \infty, z \in \gamma} \frac{\log |A(z)|}{\log |z|}=+\infty,
$$

and it follows at once from (2) that $E(z)$ tends to 0 as $z$ tends to infinity on $\gamma$.

\section{Polynomial coefficients}

Lemma 5.1. Suppose that $n$ is a non-negative integer and $A(z)=a_{n} z^{n}+\ldots+a_{0}$ is a polynomial of degree $n$, with the coefficients $a_{j}$ complex numbers. Suppose that $E$ is the product $E=f_{1} f_{2}$ of linearly independent solutions $f_{j}$ of (1), normalized so that $W\left(f_{1}, f_{2}\right)=1$. Finally, suppose that there exist constants $b \neq 0$ and $c>0$ such that $E(z)=b$ implies that $\left|E^{\prime}(z)\right| \leq c$. Then $n=0$.

Proof. Suppose that $n>0$. It is well known that $E$ has order $(n+2) / 2$, as may be seen by applying the Wiman-Valiron theory [9] to the first equation of (2). If the equation $E(z)=b$ has finitely many solutions, then $E(z)=P(z) e^{Q(z)}+b$, with $P, Q$ polynomials, and $Q$ of degree $(n+2) / 2$. But then $E(z)=0$ has infinitely many solutions, with $E^{\prime}(z)=-b\left(P^{\prime}(z) / P(z)+Q^{\prime}(z)\right) \neq O(1)$, and this is impossible since $E$ is a Bank-Laine function.

We suppose henceforth that the equation $E(z)=b$ has infinitely many solutions, and may assume that infinitely many of these lie in the region $S_{0}$ given by

$$
S_{0}=\left\{z: \quad|z| \geq R_{0}>0, \quad\left|\arg z-\theta_{0}\right| \leq \pi /(n+2)\right\},
$$


in which $\theta_{0}$ is real and satisfies $\arg a_{n}+(n+2) \theta_{0}=0(\bmod 2 \pi)$, so that $\arg z=\theta_{0}$ is a critical ray for (11) [11]. By Hille's asymptotic method [11], the equation (1) has principal solutions $u_{1}, u_{2}$ in $S_{0}$ satisfying

$$
u_{j}(z)=A(z)^{-1 / 4}(1+o(1)) \exp \left((-1)^{j} i Z\right)
$$

and

$$
u_{j}^{\prime}(z)=(-1)^{j} i A(z)^{1 / 4}(1+o(1)) \exp \left((-1)^{j} i Z\right)
$$

with

$$
Z=\int_{R_{0} e^{i \theta_{0}}}^{z} A(t)^{1 / 2} d t=\frac{2 a_{n}^{1 / 2}}{n+2} z^{(n+2) / 2}(1+o(1)) .
$$

Obviously, there exist complex numbers $B_{1}, B_{2}, B_{3}$, not all 0 , such that

$$
E=B_{1} u_{1}^{2}+B_{2} u_{1} u_{2}+B_{3} u_{2}^{2}
$$

and so, in $S_{0}$, using (40), (41) and (42),

$$
E(z)=A(z)^{-1 / 2}\left(B_{1} e^{-2 i Z}(1+o(1))+B_{2}(1+o(1))+B_{3} e^{2 i Z}(1+o(1))\right)
$$

and

$$
E^{\prime}(z)=-2 i B_{1} e^{-2 i Z}(1+o(1))+o(1)+2 i B_{3} e^{2 i Z}(1+o(1))
$$

It follows from (43) that there exists $d>0$ such that if $z_{1}$ is in $S_{0}$, with $\left|z_{1}\right|$ large and $E\left(z_{1}\right)=b$ then

$$
\max \left\{\left|B_{1} e^{-2 i Z\left(z_{1}\right)}\right|,\left|B_{3} e^{2 i Z\left(z_{1}\right)}\right|\right\}>d\left|A\left(z_{1}\right)\right|^{1 / 2} .
$$

But then (44) gives $\left|E^{\prime}\left(z_{1}\right)\right|>2 c$ if $\left|z_{1}\right|$ is large enough, and this contradiction proves the lemma.

\section{Proof of Theorem 1.3 Preliminaries}

Let $b_{1}$ and $b_{2}$ be non-zero complex numbers. Let $g$ be a transcendental entire function such that $g(z)=1$ implies that $g^{\prime}(z)= \pm b_{1}$, and $g(z)=-1$ implies that $g^{\prime}(z)= \pm b_{2}$. Set

$$
H=\frac{\left(g^{\prime}\right)^{2}+\lambda(g-1)-b_{1}^{2}}{g^{2}-1}, \quad b_{2}^{2}-2 \lambda-b_{1}^{2}=0 .
$$

Then $H$ is an entire function, and applying the Wiman-Valiron theory 9 ] to $g$ shows that $H \not \equiv 0$. Also

$$
T(r, H)=m(r, H) \leq m(r, 1 /(g-1))+m(r, 1 /(g+1))+s(r, g),
$$

in which $s(r, g)$ denotes any quantity which is $o(T(r, g))$ as $r$ tends to infinity outside a set of finite logarithmic measure. Differentiating (45) gives

$$
g^{\prime}\left(2 g^{\prime \prime}+\lambda-2 g H\right)=H^{\prime}\left(g^{2}-1\right)
$$

from which we see at once that if $H$ is a non-zero constant then $g$ has the form (5). Assuming henceforth that $H$ is non-constant we have, since $g^{\prime}(z)=0$ implies that $g(z) \neq \pm 1$,

$$
N\left(r, 1 / g^{\prime}\right) \leq N\left(r, 1 / H^{\prime}\right) \leq T(r, H)+s(r, H)
$$

and, using (46),

$$
N\left(r, 1 / g^{\prime}\right)+N(r, 1 /(g-1))+N(r, 1 /(g+1)) \leq 2 T(r, g)+s(r, g) .
$$


We apply Ahlfors' theory of covering surfaces to $g$, using the notation of [8] pp.144-149]. Let $0<\varepsilon<1 / 2$ and

$$
S(r)=\frac{1}{\pi} \int_{0}^{r} \int_{0}^{2 \pi} \frac{t\left|g^{\prime}\left(t e^{i \theta}\right)\right|^{2}}{\left(1+\left|g\left(t e^{i \theta}\right)\right|^{2}\right)^{2}} d \theta d t, \quad L(r)=\int_{0}^{2 \pi} \frac{r\left|g^{\prime}\left(r e^{i \theta}\right)\right|}{1+\left|g\left(r e^{i \theta}\right)\right|^{2}} d \theta
$$

for $r>0$. Since $g$ has no poles, [8, Theorem 5.5] implies that there is a positive constant $h$ depending only on $\varepsilon$ such that

$$
S(r) \leq n(r)-n_{1}(r)+h L(r) \quad \text { for } \quad r>0 .
$$

Here $n(r)$ denotes the total multiplicity of all the islands [8, p.145] for $g$ over $B( \pm 1, \varepsilon)$ in $B(0, r)$, and $n_{1}(r)$ denotes the sum of the excesses [8, p.147] of these islands. If an island has multiplicity $p$ and connectivity $q$ (each necessarily at least $1)$, then the excess of the island is $(p-1)+(q-1)$. Let $n^{s}(r)$ count the number of simple islands over $B( \pm 1, \varepsilon)$ in $B(0, r)$, and let $n^{m}(r)$ and $n_{1}^{m}(r)$ be the total multiplicity and total excess of all islands in $B(0, r)$ of multiplicity at least 2 .

By (49), the Ahlfors-Shimizu characteristic $T_{0}(r, g)$ [8, p.12] satisfies

$$
T_{0}(r, g) \leq N(r)-N_{1}(r)+h \int_{0}^{r} \frac{L(t) d t}{t}, \quad N(r)=\int_{0}^{r} \frac{n(t) d t}{t} .
$$

We define $N_{1}(r), N^{s}(r), N^{m}(r), N_{1}^{m}(r)$ in the same way as $N(r)$. Further, by [19] and the fact [8, p.13] that $T_{0}(r, g)=T(r, g)+O(1)$, we have

$$
\int_{0}^{r} \frac{L(t) d t}{t}=s(r, g) \text {. }
$$

Lemma 6.1. We have

$$
T(r, g) \leq 2 N^{s}(r)+s(r, g) .
$$

Proof. If an island $D$ over $B( \pm 1, \varepsilon)$ has multiplicity $p \geq 2$ and connectivity $q$, then the excess of $D$ is

$$
(p-1)+(q-1) \geq p-1=p(1-1 / p) \geq p / 2 .
$$

Thus $N^{m}(r) \leq 2 N_{1}^{m}(r)$ and (50) and [51] give

$$
2 T(r, g) \leq 2 N^{s}(r)+N^{m}(r)+s(r, g) .
$$

Next, if an island $D^{\prime}$ has multiplicity $p^{\prime} \geq 1$ then one of the equations $g(z)= \pm 1$ has $p^{\prime}$ solutions in $D^{\prime}$. Hence

$$
n^{s}(r)+n^{m}(r) \leq n(r, 1 /(g-1))+n(r, 1 /(g+1))
$$

and (53) gives

$$
2 T(r, g) \leq N^{s}(r)+N(r, 1 /(g-1))+N(r, 1 /(g+1))+s(r, g) .
$$

Combining (47) and (54) we get

$$
2 T(r, g)+N\left(r, 1 / g^{\prime}\right) \leq N^{s}(r)+2 T(r, g)+s(r, g)
$$

and so

$$
N\left(r, 1 / g^{\prime}\right) \leq N^{s}(r)+s(r, g) .
$$

Suppose again that $D$ is an island of multiplicity $p \geq 2$ and connectivity $q$. Then by the Riemann-Hurwitz formula [26, p.7], the number $y$ of critical points of $g$ in $D$, counting multiplicity, satisfies

$$
q-2=p(-1)+y, \quad y=p+q-2 \geq p-1=p(1-1 / p) \geq p / 2 .
$$


Thus (55) gives

$$
N^{m}(r) \leq 2 N\left(r, 1 / g^{\prime}\right) \leq 2 N^{s}(r)+s(r, g),
$$

which, on substitution into (53), gives (52). Lemma 6.1 is proved.

Next, set

$$
E_{1}=\frac{g-1}{b_{1}}, \quad E_{2}=\frac{g+1}{b_{2}} .
$$

Then $E_{1}, E_{2}$ are Bank-Laine functions. As in Section 10 define entire functions $A_{1}, A_{2}$ by

$$
4 A_{j}=\left(E_{j}^{\prime} / E_{j}\right)^{2}-2 E_{j}^{\prime \prime} / E_{j}-1 / E_{j}^{2}
$$

Lemma 6.2. Let $d= \pm 1$. There exists a positive constant $c_{1}$, depending only on $b_{1}$ and $b_{2}$, such that if $g$ maps the domain $D$ univalently onto $B(d, 1 / 4)$, then $D^{*}=\{z \in D: 1 / 8<|g(z)-d|<1 / 4\}$ has area $A^{*} \geq c_{1}$.

Proof. The function $G=4(g-d)$ maps $D$ univalently onto $B(0,1)$, and we denote by $\psi(w)$ the inverse function $G^{-1}(w)$. The Koebe distortion theorem [21, p.9] gives $\left|\psi^{\prime}(w)\right| \geq c_{2}$ for $1 / 2 \leq|w| \leq 3 / 4$, in which $c_{2}$ is a positive constant depending only on $b_{1}$ and $b_{2}$. Lemma 6.2 now follows at once from the standard formula for $A^{*}$ as the integral of $\left|\psi^{\prime}(w)\right|^{2}$.

Lemma 6.3. We have

$$
T(r, g)=O\left(r^{2}\right), \quad r \rightarrow \infty .
$$

Proof. We have, using Lemma 6.2.

$$
n^{s}(r)=O\left(r^{2}\right), \quad N^{s}(r)=O\left(r^{2}\right)
$$

and the result follows using Lemma 6.1.

Proposition 6.1. At least one of $A_{1}, A_{2}$ is a polynomial.

The proof of Proposition 6.1 will require the remainder of this section. Assume that $A_{1}, A_{2}$ are both transcendental. Since $E_{1}, E_{2}$ have finite order, by Lemma 6.3, each $E_{j}$ has asymptotic value 0 , using Theorem 1.4, and so 1 and -1 are asymptotic values of $g$. Further, we may choose positive constants $M_{1}, M_{2}$ and a sequence $v_{q} \rightarrow \infty$ such that

$$
\left|E_{j}^{\prime}(z) / E_{j}(z)\right|^{2}+2\left|E_{j}^{\prime \prime}(z) / E_{j}(z)\right| \leq|z|^{M_{1}}, \quad j=1,2,
$$

for all $z$ with $|z|>2$ and lying outside the set

$$
V=\bigcup_{q=1}^{\infty} B\left(v_{q},\left|v_{q}\right|^{-M_{2}}\right), \quad \sum_{q=1}^{\infty}\left|v_{q}\right|^{-M_{2}}<\infty .
$$

By (56), (57) and (59), there exists $M_{3}>4$ such that if $|z|>M_{3}$ and $z$ lies outside $V$ with $\left|A_{j}(z)\right|>|z|^{M_{1}+1}$, then $E_{j}(z)$ is small and $\left|g(z)+(-1)^{j}\right|<1 / 8$.

Choose

$$
M_{4}>M\left(M_{3}, A_{1}\right)+M\left(M_{3}, A_{2}\right)+M\left(M_{3}, g\right)+2
$$

and, for $j=1,2$, let $D_{j}$ be an unbounded component of the set $\left\{z:\left|A_{j}(z) z^{-M_{1}-1}\right|>\right.$ $\left.M_{4}\right\}$, such a $D_{j}$ existing since $A_{j}$ is assumed transcendental. By the choice of $M_{3}, M_{4}$, the domains $D_{1}, D_{2}$ lie in $|z|>M_{3}$ and $D_{1} \cap D_{2} \subseteq V$. 
Since $g$ has asymptotic values $1,-1$, there exist at least two components $D_{3}, D_{4}$ of the set $\left\{z:|g(z)|>M_{4}\right\}$, both lying in $|z|>M_{3}$, and $D_{3} \cap\left(D_{1} \cup D_{2}\right) \subseteq$ $V, D_{4} \cap\left(D_{1} \cup D_{2}\right) \subseteq V$, again by the choice of $M_{3}, M_{4}$.

For $t \geq M_{3}$, let $\theta_{j}(t)$ be the angular measure of the intersection of $D_{j}$ with the circle $S(0, t)$. Define $\theta_{j}^{*}(t)$ to equal $\theta_{j}(t)$, except that $\theta_{j}^{*}(t)=\infty$ if the whole circle $S(0, t)$ lies in $D_{j}$. There exists $M_{5}>0$ such that $\theta_{j}(t)>0$ for each $j$ and for all $t \geq M_{5}$. Since the intersection of $D_{j}$ and $D_{j^{\prime}}$, for $j \neq j^{\prime}$, lies in $V$, we have $\theta_{j}(t)=\theta_{j}^{*}(t)$ for all $t$ outside a set $F_{0}$ of finite measure. Hence, by Lemma 6.3 and a standard application of Lemma 2.2. we have

$$
\int_{M_{5}}^{r} \frac{\pi d t}{t \theta_{j}(t)} \leq \int_{M_{5}}^{r} \frac{\pi d t}{t \theta_{j}^{*}(t)}+O(1) \leq 2 \log r+O(1)
$$

as $r \rightarrow \infty$.

Let $\theta(t)=\pi$ for $t \in F_{0}$, and let

$$
\theta(t)=2 \pi-\sum_{j=1}^{4} \theta_{j}(t) \geq 0, \quad t \notin F_{0} .
$$

For $t \notin F_{0}$ a standard application of the Cauchy-Schwarz inequality gives

$$
16 \leq(2 \pi-\theta(t)) \sum_{j=1}^{4} \frac{1}{\theta_{j}(t)} \leq 2 \pi \sum_{j=1}^{4} \frac{1}{\theta_{j}(t)},
$$

and so

$$
8 \log r-O(1) \leq \sum_{j=1}^{4} \int_{M_{5}}^{r} \frac{\pi d t}{t \theta_{j}(t)}
$$

Combining (61) and we we that, for each $j$,

as $r \rightarrow \infty$.

$$
\int_{M_{5}}^{r} \frac{\pi d t}{t \theta_{j}(t)}=2 \log r+O(1)
$$

Lemma 6.4. $g$ has order 2, mean type.

Proof. Lemma 6.4 follows at once from Lemma 6.3 and 65).

We now have, as $r \rightarrow \infty$, using (63) again,

$$
\sum_{j=1}^{4} \int_{M_{5}}^{r} \frac{\pi d t}{t \theta_{j}(t)} \geq \int_{M_{5}}^{r} \frac{16 \pi d t}{t(2 \pi-\theta(t))}-O(1) \geq 8 \log r-O(1)+\int_{M_{5}}^{r} \frac{4 \theta(t) d t}{\pi t}
$$

and hence, using (65),

$$
\int_{M_{5}}^{r} \frac{\theta(t) d t}{t}=O(1)
$$

It follows that if the positive integer $n$ is large, then

and so

$$
\int_{2^{n}}^{2^{n+1}} t \theta(t) d t \leq 4^{n+1} \int_{2^{n}}^{2^{n+1}} \frac{\theta(t) d t}{t}=o\left(4^{n}\right)
$$

$$
\int_{1}^{r} t \theta(t) d t=o\left(r^{2}\right)
$$

as $r \rightarrow \infty$. 
Now suppose that $d= \pm 1$ and $r$ is large, and that $D$ is a simple island for $g$ over $B(d, 1 / 4)$, lying in $M_{5}<|z|<r$. Then $D$ obviously cannot meet $D_{3}$ or $D_{4}$, and $D^{*}=\{z \in D: 1 / 8<|g(z)-d|<1 / 4\}$ has $D^{*} \cap D_{1} \subseteq V, D^{*} \cap D_{2} \subseteq V$. Since $D^{*}$ has area $A^{*} \geq c_{1}$, by Lemma [6.2, and the sum of $A^{*}$ over all such $D$ is at most

$$
2 \int_{1}^{r} t \theta(t) d t+O(1)
$$

we obtain from (66),

$$
n^{s}(r)=o\left(r^{2}\right), \quad N^{s}(r)=o\left(r^{2}\right),
$$

and so $T(r, g)=o\left(r^{2}\right)$, using Lemma 6.1] This contradicts Lemma 6.4, and proves Proposition 6.1.

\section{Proof of Theorem 1.3}

Suppose that $g$ is as in the hypotheses. Applying a linear transformation we may assume without loss of generality that $a_{1}=1, a_{2}=-1$. Thus the functions $E_{1}, E_{2}$ defined by (56) are Bank-Laine functions, and $A_{1}, A_{2}$ as defined by (57) are entire.

If $g$ is a non-constant polynomial then so is $E_{1}$, and it follows at once from (57) that $A_{1} \equiv 0$. Thus $E_{1}$ has degree at most 2 and so has $g$. We assume henceforth that $g$ is transcendental.

The function $g$ thus satisfies the assumptions of Section 6, and by Proposition 6.1 at least one of $A_{1}, A_{2}$, say $A_{1}$, is a polynomial. But then, by Lemma 5.1 $A_{1}$ must be constant, and (5) follows at once.

\section{Proof of Theorem 1.2}

Suppose that $f$ and $g$ are transcendental, with $g$ entire and $f$ meromorphic in the plane, and that the composition $E=f \circ g$ is a Bank-Laine function of finite order. Define the entire function $A$ by (2).

Then $f$ has order 0 [8, p.53], and consequently has infinitely many zeros. It follows from Theorem [1.1 that $g$ has the form (5), without loss of generality with $b=1 / 2$, so that $E$ and $A$ have period $2 \pi i$. We may therefore write

$$
E(z)=F\left(e^{z}\right)=F_{1}\left(e^{z}\right)+F_{2}\left(e^{-z}\right), \quad A(z)=B\left(e^{z}\right)=B_{1}\left(e^{z}\right)+B_{2}\left(e^{-z}\right),
$$

in which $F_{1}, F_{2}, B_{1}, B_{2}$ are entire functions.

Lemma 8.1. $F_{1}, F_{2}, B_{1}, B_{2}$ have order 0 .

Proof. Let $\rho$ be large and positive, and choose a real $t$ with $\left|F_{1}\left(\rho e^{i t}\right)\right|=M\left(\rho, F_{1}\right)$. Next, choose $z_{0}=\log \rho+i s,-\pi \leq s \leq \pi$, such that $e^{z_{0}}=\rho e^{i t}$. We then have

$$
\begin{aligned}
M\left(\rho, F_{1}\right) & =\left|F\left(\rho e^{i t}\right)\right|+O(1)=\left|E\left(z_{0}\right)\right|+O(1) \\
& \leq \exp \left(c_{1}\left|z_{0}\right|^{M_{1}}\right) \leq \exp \left(c_{2}(\log \rho)^{M_{1}}\right),
\end{aligned}
$$

for some positive constants $c_{1}, c_{2}, M_{1}$ independent of $\rho$.

The proof of Theorem 1.2 now proceeds using ideas from the theory of periodic differential equations [2]. Since (11) has linearly independent solutions each having zeros with finite exponent of convergence, [4, Theorem 4] shows that $B$ is a rational function. Substituting $E(z)=F\left(e^{z}\right), A(z)=B\left(e^{z}\right)$ into the second equation of (2) gives a third order linear differential equation for $F$, with coefficients which 
are rational functions. Since $F$ has order 0 , a standard application of the WimanValiron theory [9] shows that $F$ is a rational function, and so is $f$. This contradiction proves the theorem.

\section{REFERENCES}

1. S. Bank and I. Laine, On the oscillation theory of $f^{\prime \prime}+A f=0$ where $A$ is entire, Trans. Amer. Math. Soc. 273 (1982), 351-363. MR 83k:34009

2. S. Bank and I. Laine, Representations of solutions of periodic second order linear differential equations, J. reine angew. Math. 344 (1983), 1-21. MR 85a:34008

3. S. Bank and I. Laine, On the zeros of meromorphic solutions of second-order linear differential equations, Comment. Math. Helv. 58 (1983), 656-677. MR 86a:34008

4. S. Bank and J.K. Langley, Oscillation theorems for higher order linear differential equations with entire periodic coefficients, Comment. Math. Univ. St. Paul. 41 (1992), 65-85. MR 93e:34011

5. W. Bergweiler, Proof of a conjecture of Gross concerning fixpoints, Math. Zeit. 204 (1990), 381-390. MR 92j:30028

6. R. Goldstein, On factorization of certain entire functions, J. London Math. Soc. (2) 2 (1970), 221-224. MR 41:2012

7. F. Gross, Factorization of meromorphic functions, Mathematics Research Center, Naval Research Lab., Washington D.C., 1972. MR 53:11030

8. W.K. Hayman, Meromorphic functions, Oxford at the Clarendon Press, 1964. MR 29:1337

9. W.K. Hayman, The local growth of power series: a survey of the Wiman-Valiron method, Canad. Math. Bull. 17 (1974), 317-358. MR 52:5965

10. W.K. Hayman, On Iversen's theorem for meromorphic functions with few poles, Acta Math. 141 (1978), 115-145. MR 58:11409

11. E. Hille, Ordinary differential equations in the complex domain, Wiley, New York, 1976. MR 58:17266

12. I. Laine, Nevanlinna theory and complex differential equations, de Gruyter Studies in Math. 15, Walter de Gruyter, Berlin/New York 1993. MR 94d:34008

13. J.K. Langley, On complex oscillation and a problem of Ozawa, Kodai Math. J. 9 (1986), 430-439. MR 87j:34018

14. J.K. Langley, Quasiconformal modifications and Bank-Laine functions, Archiv der Math. 71 (1998), 233-239. MR 99e:34001

15. J.K. Langley, Bank-Laine functions with sparse zeros, Proc. Amer. Math. Soc. 129 (2001), 1969-1978. CMP 2001:11

16. J.K. Langley and D.F. Shea, On multiple points of meromorphic functions, J. London Math. Soc. (2) 57 (1998), 371-384. MR 99g:30043

17. J. Lewis, J. Rossi and A. Weitsman, On the growth of subharmonic functions along paths, Ark. Mat. 22 (1983), 104-114. MR 85f:31002

18. Linear and complex analysis problem book, edited by V.P. Havin, S.V. Khrushchev and N.K. Nikol'skii, Lecture Notes in Mathematics 1043, Springer-Verlag, Berlin-New York, 1984. MR 85k:46001

19. J. Miles, A note on Ahlfors' theory of covering surfaces, Proc. Amer. Math. Soc. 21 (1969), 30-32. MR 39:450

20. J. Miles and J. Rossi, Linear combinations of logarithmic derivatives of entire functions with applications to differential equations, Pacific J. Math. 174 (1996), 195-214. MR 97e:30055

21. C. Pommerenke, Boundary behaviour of conformal maps, Grundlehren der Mathematischen Wissenschaften 299, Springer, Berlin 1992. MR 95b:30008

22. J. Rossi, The length of asymptotic paths of harmonic functions, J. London Math. Soc. 30 (1984), 73-78. MR 86d:31001

23. J. Rossi, Second order differential equations with transcendental coefficients, Proc. Amer. Math. Soc. 97 (1986), 61-66. MR 87f:30078

24. L.C. Shen, Solution to a problem of S. Bank regarding the exponent of convergence of the solutions of a differential equation $f^{\prime \prime}+A f=0$, Kexue Tongbao 30 (1985), 1581-1585. MR 87j:34020

25. L.C. Shen, Construction of a differential equation $y^{\prime \prime}+A y=0$ with solutions having prescribed zeros, Proc. Amer. Math. Soc. 95 (1985), 544-546. MR 87b:34005 
26. N. Steinmetz, Rational iteration, de Gruyter Studies in Mathematics 16, Walter de Gruyter, Berlin/New York, 1993. MR 94h:30035]

27. M. Tsuji, Potential theory in modern function theory, Maruzen, Tokyo, 1959. MR 22:5712

School of Mathematical Sciences, University of Nottingham, Nottingham NG7 2RD, $\mathrm{UK}$

E-mail address: jkl@maths.nott.ac.uk 